TRANSACTIONS OF THE

AMERICAN MATHEMATICAL SOCIETY

Volume 333, Number 2, October 1992

\title{
RADII OF CONVERGENCE AND INDEX FOR $p$-ADIC DIFFERENTIAL OPERATORS
}

\author{
PAUL THOMAS YOUNG
}

\begin{abstract}
We study the radii of $p$-adic convergence of solutions at a generic point of homogeneous linear differential operators whose coefficients are analytic elements. As an application we prove a conjecture of P. Robba (for a certain class of operators) concerning the relation between radii of convergence and index on analytic elements. We also give an explicit factorization theorem for $p$-adic differential operators, based on the radii of generic convergence and the slopes of the associated Newton polygon.
\end{abstract}

\section{INTRODUCTION}

Motivated by the recent results of $P$. Robba $[10,11]$ relating the index of $p$-adic linear differential operators to the generic radius of convergence of their homogeneous solutions, in this work we make a detailed study of the radii of $p$-adic convergence of such solutions at a generic point. We then use the information obtained to verify that the index-radius formula conjectured by Robba $[10, \S 4.13]$ does indeed hold for a certain class of operators. In addition, we use the correspondence between radii of convergence of solutions and slopes of the associated Newton polygon to describe an explicit factorization of linear differential operators, which is an extension of the factorizations given in [5]. We conclude with several other applications, demonstrating in particular how these results give information concerning the description of solutions unbounded in the generic disk and of solutions at irregular singular points.

It has been known for some time $[5,8]$ that there is a factorization of linear differential operators corresponding to the filtration of the solution space near the generic point according to radius of convergence and growth conditions. B. Dwork and P. Robba have also shown that there is a factorization related to the associated Newton polygon $[5, \S 6.2 .3 .3]$. However, this latter result treated only the first side of the Newton polygon, and the relation between these two factorizations remained unclear; furthermore, there have been no general methods for determining the precise radii of convergence of solutions at a generic point, or for determining when distinct radii of convergence will occur. In addition to giving exact formulae for the radii of convergence, the present work also provides a connection between several objects associated to a $p$-adic differential operator, in particular, the index, the radii of convergence of solutions at the generic point, and the Newton polygon. Our main result (Theorem 3.1 below)

Received by the editors January 27, 1989 and, in revised form, August 1, 1990.

1980 Mathematics Subject Classification (1985 Revision). Primary 12H25, 12B40. 
gives the relationship between the slopes of the Newton polygon and the radii of convergence of solutions at generic points. This result is the first we know of that gives formulae for the exact radii of generic convergence of solutions for such a wide class of operators, without any hypothesis on the existence of Frobenius structures. In Corollary 4.1 we reiterate this result in terms of the factorizations of the operator, providing the connection between the factorizations of $\S \S 4$ and 6 of [5]. Corollary 4.3 shows the relationship between radius of generic convergence and index on analytic elements.

In [12] Robba has conjectured a formula for the index on $\mathscr{H}_{\infty}^{\dagger}(1)^{k+1}$ of the $k$ th symmetric power of the $p$-adic Bessel operator, based on a conjecture concerning the effect of ramification of the variable on the index $[11, \S 8.3]$. In [13] we have used the methods found in this paper to show that for the odd symmetric powers of the Bessel operator, this latter conjecture is indeed true relative to sufficiently small disks about the irregular singular point at $\infty$. We believe that these methods and the methods of Robba [11] will allow us to extend this result to the even symmetric powers as well. We intend to return to this question in a future paper.

The results of this paper represent a portion of the author's doctoral dissertation [13]. The reader is referred to [13] for more complete details and additional remarks. The author would also like to thank A. Adolphson for many helpful conversations during the entire course of this work.

\section{NOTATION AND PRELIMINARIES}

Let $K$ be an algebraically closed field of characteristic zero, complete under a nonarchimedean valuation, ord, which is normalized so that ord $p=1$, where $p>0$ is the characteristic of the residue class field of $K$. We imbed $K$ in an extension field $\Omega$ which is algebraically closed, complete under a valuation extending that of $K$, linearly disjoint from $K(x)$ over $K$, and whose valuation ring contains a unit $t$ whose image $\bar{t}$ in the residue class field is transcendental over the residue class field of $K$. We also suppose that the absolute value thus induced on $\Omega$ by ord is normalized so that $|p|=p^{-1}$; therefore if $x \in \Omega$ is such that $|x|=r$, then ord $x=-\log r$, where $\log$ refers to the usual real logarithm to the base $p$. Also, throughout this paper we will adopt a fairly standard notation, using $\pi$ to denote a solution in $K$ to $\pi^{p-1}=-p$.

Let

$$
\left|\Omega^{*}\right|=\{|x|: x \in \Omega \backslash\{0\}\}
$$

be the multiplicative group of values of $\Omega^{*}$. By our hypotheses $\left|\Omega^{*}\right|$ is dense in $\mathbb{R}^{+}$. For $a \in \Omega$ and $r \in\left|\Omega^{*}\right|$ we define the circumferenced and uncircumferenced disks

$$
\begin{aligned}
& B\left(a, r^{+}\right)=\{x \in \Omega:|x-a| \leq r\}, \\
& B\left(a, r^{-}\right)=\{x \in \Omega:|x-a|<r\} .
\end{aligned}
$$

If $c \in K$, we say that $t \in \Omega$ is a $(c, r)$-generic point if $|t-c|=r>0$ and $B\left(t, r^{-}\right) \cap K=\varnothing$. The disk $B\left(t, r^{-}\right)$is called a $(c, r)$-generic disk. (In Robba's terminology [10], $t$ is called a generic point on the circumference $C(c, r)$, and $B\left(t, r^{-}\right)$is called a generic disk of the circumference $C(c, r)$.) By our hypotheses on $\Omega$ there exist $(c, r)$-generic points for every $c \in K$ and every $r \in\left|\Omega^{*}\right|$. 
We now define, for each $c \in K$ and $r \in \mathbb{R}^{+}$, an absolute value $|\cdot|_{c}(r)$ on $K(x)$ as follows: For $f \in K[x]$, write $f=\sum a_{i}(x-c)^{i}$ and define

$$
|f|_{c}(r)=\sup _{i}\left|a_{i}\right| r^{i}
$$

We extend the definition of $|\cdot|_{c}(r)$ to rational functions $h=g / f$ by setting

$$
|h|_{c}(r)=|g|_{c}(r) /|f|_{c}(r)
$$

where $f, g \in K[x]$. It is well known that this definition is independent of the choice of $g, f$, and that in fact $|h|_{c}(r)=|h(t)|$ for all $h \in K(x)$ and all $(c, r)$-generic points $t$. We define $E_{c, r}$ to be the completion of $K(x)$ under the norm $|\cdot|_{c}(r)$; the $K$-algebra $E_{c, r}$ is then a (nonarchimedean) Banach space over $K$. Note that the field $E_{0,1}$ is precisely the field $E$ described in [5].

A subset $A$ of $\mathbb{P}(\Omega)$ is said to be a $(c, r)$-very standard set if $A$ is a union of sets which are either of the form $B\left(a_{i}, r^{-}\right)$with $\left|a_{i}-c\right| \leq r$, or the set $B\left(c, r^{+}\right)^{c}$. (In [5], Dwork and Robba have used the term "very standard set" to describe what would be called a " $(0,1)$-very standard set" in our terminology.)

If $A$ is a subset of $\mathbb{P}(\Omega)$ with $d\left(A, A^{c}\right)>0$ (in particular, if $A$ is a very standard set), we define $R(A)$ to be the set of all $f \in K(x)$ which as functions on $\mathbb{P}(\Omega)$ have no poles on $A$. A function $f: A \rightarrow \Omega$ is an analytic element on $A$ if it is the uniform limit on $A$ of a sequence in $R(A)$; we denote the set of analytic elements on $A$ by $H(A)$. The $K$-algebra $H(A)$ is then a (nonarchimedean) Banach space over $K$ under the supremum norm $\|f\|_{A}=\sup _{x \in A}|f(x)|$. The advantage of working with very standard sets is that if $A$ is a $(c, r)$-very standard set and $f \in H(A)$, then $|f|_{c}(r)=\|f\|_{A}$; this is easily verified for $f \in R(A)$ and extended to $f \in H(A)$ by continuity.

If $A$ is a subset of $\mathbb{P}(\Omega)$ with $d\left(A, A^{c}\right)>0$, we define $M(A)$, the field of meromorphic elements on $A$, to be the field of quotients of the integral domain $H(A)$. We extend the absolute values $\|\cdot\|_{A}$ and $|\cdot|_{c}(r)$ to $M(A)$ in the natural way: for $h=g / f$ we set

$$
\|h\|_{A}=\|g\|_{A} /\|f\|_{A}, \quad|h|_{c}(r)=|g|_{c}(r) /|f|_{c}(r) .
$$

If $A$ contains a $(c, r)$-very standard set, then we have natural inclusions $H(A)$ $\subseteq M(A) \subseteq E_{c, r}$.

If $A \subseteq \mathbb{P}(\Omega)$ satisfies $d\left(A, A^{c}\right)>0$ and $h \in K(x)$, we define $\operatorname{ord}_{A} h$ to be the number of zeros of $h$ on $A$ minus the number of poles of $h$ on $A$, counted with multiplicity. As conventions we will also write

$$
\begin{aligned}
\operatorname{ord}_{a}^{+}(h, r) & =\operatorname{ord}_{B\left(a, r^{+}\right)} h, \\
\operatorname{ord}_{a}^{-}(h, r) & =\operatorname{ord}_{B\left(a, r^{-}\right)} h, \\
\operatorname{ord}_{a} h & =\operatorname{ord}_{\{a\}} h .
\end{aligned}
$$

If $A$ is a $(c, r)$-very standard set and $h \in H(A), h \neq 0$, then $h$ has only finitely many zeros on $A$ (again by a straightforward continuity argument from $R(A)$ to $H(A))$. It therefore makes sense for us to extend the definition of $\operatorname{ord}_{A} h$ to the case where $h$ is a meromorphic element on a very standard set $A$ by setting $\operatorname{ord}_{A} h=\operatorname{ord}_{A} g-\operatorname{ord}_{A} f$, where $h=g / f$ with $g, f \in H(A)$.

For linear operators $L: H(A) \rightarrow H(A)$ we have the operator norm

$$
\|L\|_{A}=\sup _{h \neq 0}\|L h\|_{A} /\|h\|_{A}
$$


and if $A$ is a $(c, r)$-very standard set we may also define

$$
\|L\|_{c, r}=\sup _{h \neq 0}|L h|_{c}(r) /|h|_{c}(r) .
$$

Again, if $A$ is a $(c, r)$-very standard set then $\|\cdot\|_{c, r}=\|\cdot\|_{A}$.

If $V$ is a vector space and $L: V \rightarrow V$ is a linear transformation, then $L$ is said to have an index on $V$ if the kernel and cokernel of $L$ are both finite dimensional; in this case the index of $L$ on $V$ is defined to be

$$
\chi(L ; V)=\operatorname{dim} \operatorname{ker} L-\operatorname{dim} \operatorname{coker} L .
$$

The reader is referred to $[8,9]$ for some of the basic properties of indices. In addition, we will also need the following propositions.

Proposition 2.1. If $A$ is $a(c, r)$-very standard set and $f \in H(A), f \neq 0$, then multiplication by $f$ is injective and has index $\chi(f ; H(A))=-\operatorname{ord}_{A} f$, and has a continuous left inverse $\varphi: H(A) \rightarrow H(A)$ with $\|\varphi\|_{c, r} \leq|f|_{c}(r)^{-1}$.

Proof. Robba has already proved these results in the case where $f \in R(A)$ (see [9, Lemma 3.3; 11, Lemme 3.4]). The proof for elements of $H(A)$ may be obtained from these results with the aid of [8, Lemmas 4.3 and 4.4], and the fact that nonzero elements of $H(A)$ have only finitely many zeros on $A$.

Proposition 2.2. The formal derivative map $D=(d / d x): K(x) \rightarrow K(x)$ extends uniquely to a continuous map (also denoted by $D$ ) from $E_{c, r}$ to itself. Furthermore, for each $m \in \mathbb{Z}^{+}$and $f \in E_{c, r}$ we have

$$
\left|f^{(m)}\right|_{c}(r) \leq r^{-m}|m !| \cdot|f|_{c}(r)
$$

Proof. This is a simple modification of the proof of Proposition 2.1.8 of [2].

It follows that if $A$ is a $(c, r)$-very standard set then the derivative map may be extended to a map $D: M(A) \rightarrow M(A)$. If $A$ is a $(c, r)$-very standard set we define $\mathfrak{G}_{A}$ (resp. $\mathfrak{R}_{A}$ ) to be the (noncommutative) Euclidean ring $H(A)[D]$ (resp. $M(A)[D])$, which we identify naturally with the ring of linear differential operators with coefficients in $H(A)$ (resp. $M(A)$ ). We also define $\mathfrak{R}_{c, r}$ to be the ring $E_{c, r}[D]$. Note that there are natural inclusions $\mathfrak{G}_{A} \subseteq \mathfrak{R}_{A} \subseteq \mathfrak{R}_{c, r}$. We also note $[8, \S 1.11]$ that if $L=\sum h_{i} D^{i} \in \mathfrak{R}_{c, r}$, then

$$
\|L\|_{c, r}=\max _{i}\left\{r^{-i}|i !| \cdot\left|h_{i}\right|_{c}(r)\right\} .
$$

We may consider the elements of $\mathfrak{R}_{c, r}$ (resp. $\mathfrak{G}_{A}$ ) as linear transformations on $E_{c, r}$ (resp. $H(A)$ ); however, as linear operators the elements of $\mathfrak{R}_{A}$ need not be stable on $H(A)$. Therefore our index results will be phrased in terms of elements of $\mathfrak{G}_{A}$, although for purposes of generality we will phrase our convergence results in terms of elements of $\mathfrak{R}_{c, r}$.

Let $L=q_{0} D^{n}+q_{1} D^{n-1}+\cdots+q_{n-1} D+q_{n}$ be a monic element of $\mathfrak{R}_{c, r}$. The primary focus of this work is to determine the exact radii of convergence $\rho(u)$ of solutions $u(x)$ of the homogeneous equation $L u=0$ which are analytic in a neighborhood of a $(c, r)$-generic point $t$. Following Robba [10], since we wish to relate this to the index of elements $L$ of $\mathfrak{G}_{A}$ on $H\left(B\left(c, r^{ \pm}\right)\right)$, we will use $\chi_{c}^{ \pm}(L, r)$ to denote this index, and for first order operators we denote by $\rho_{c}(L, r)$ the radius of convergence of the nontrivial solutions of $L u=0$ at a $(c, r)$-generic point $t$. 
To generalize this definition for $n$th order operators $L$ as above, we define

$$
\rho_{c}(L, r)=\sup \left\{\rho\left(u_{1}\right) \cdots \rho\left(u_{n}\right)\right\},
$$

where the supremum is over all sets of $n$ linearly indenendent solutions $\left\{u_{1}, \ldots, u_{n}\right\}$ of $L u=0$ at $t$. We note that by Lemma 3.3 below, the supremum is actually a maximum, since the set of possible values for the $\rho\left(u_{i}\right)$ is discrete. A basis of solutions $\mathfrak{B}=\left\{u_{1}, \ldots, u_{n}\right\}$ of $L$ at $t$ for which the maximum is attained will be called an optimal basis for the kernel of $L$ at $t$.

Our aim will be to relate the radii of convergence of the elements in an optimal basis for $L$ at $t$ to the absolute values of the roots of the polynomial $\Delta_{t}(L) \in \Omega[\lambda]$ defined by

$$
\Delta_{t}(L)(\lambda)=q_{0}(t) \lambda^{n}+q_{1}(t) \lambda^{n-1}+\cdots+q_{n-1}(t) \lambda+q_{n}(t) .
$$

Since $|h(t)|=|h|_{c}(r)$ for all $(c, r)$-generic points $t$ and all $h \in E_{c, r}$, the magnitudes of the roots of $\Delta_{t}(L)$ are independent of the choice of $t$. By means of left multiplication by $q_{0}^{-1}$ we may assume that $L$ is monic (i.e., $q_{0}=1$ ) without affecting the roots of $\Delta_{t}(L)$ or the solutions at $t$.

\section{THE CONVERGENCE THEOREM}

We now state our principal result.

Theorem 3.1. Let $L=D^{n}+q_{1} D^{n-1}+\cdots+q_{n} \in \mathfrak{R}_{c, r}$ and let $t$ be a $(c, r)$ generic point. Then there exists an optimal basis $\mathfrak{B}$ for the kernel of $L$ at $t$ and a one-to-one correspondence between the roots of $\Delta_{t}(L)$ and the elements of $\mathfrak{B}$ such that

(i) Corresponding to every root $\lambda$ of $\Delta_{t}(L)$ satisfying $|\lambda|>r^{-1}$ there is an element of $\mathfrak{B}$ which converges exactly on the disk

$$
\operatorname{ord}(x-t)>\frac{1}{p-1}+\log |\lambda|,
$$

and is bounded on this disk.

(ii) Corresponding to every root $\lambda$ of $\Delta_{t}(L)$ satisfying $|\lambda| \leq r^{-1}$ there is an element of $\mathfrak{B}$ which converges at least on the disk

$$
\operatorname{ord}(x-t)>\frac{1}{p-1}+\operatorname{ord}(t-c),
$$

and is bounded on this disk.

Before we prove this theorem we need some preliminary results.

Lemma 3.2. Let $L=D^{n}+q_{1} D^{n-1}+\cdots+q_{n} \in \mathfrak{R}_{c, r}$ and let $t$ be a $(c, r)$-generic point. Let $\rho \in(0, r]$ and define a norm $\|\cdot\|_{\rho}$ on $\mathfrak{R}_{c, r}$ by

$$
\left\|\sum a_{m} D^{m}\right\|_{\rho}=\sup _{m}\left\{|m !| \rho^{-m}\left|a_{m}\right|_{c}(r)\right\} .
$$

Then if $\|R \circ L-1\|_{\rho}$ is bounded away from zero independent of $R \in \mathfrak{R}_{c, r}$, there exist nontrivial solutions of $L$ at $t$ which converge at least on the disk $B\left(t, \rho^{-}\right)$.

Lemma 3.3. Let $L=D^{n}+q_{1} D^{n-1}+\cdots+q_{n} \in \mathfrak{R}_{c, r}$ and let $t$ be a $(c, r)$-generic point. Then for each $\rho \in(0, r]$, there exist unique monic elements $M, N \in \mathfrak{R}_{c, r}$ 
such that $L=N \circ M$ and $M$ annihilates precisely those solutions of $L$ which converge at least on the disk $B\left(t, \rho^{-}\right)$and are bounded on that disk.

Proofs. These results are generalizations of some results of Robba $[8, \S 2]$, which he proved in much greater generality for the case $c=0, r=1$. To obtain the proofs of these results for general $c$ and $r$ requires only a minor modification of Robba's proofs.

We now give the proof of Theorem 3.1 in the case $n=1$.

Lemma 3.4. Let $L \in \mathfrak{R}_{c, r}$ be the monic first-order operator $L=D+q$.

(i) If $q$ satisfies $|(x-c) q|_{c}(r)>1$, then the nontrivial solutions $u(x)$ to $L u=0$ near a $(c, r)$-generic point $t$ converge and are bounded on the disk

$$
\operatorname{ord}(x-t)>\frac{1}{p-1}+\log |q|_{c}(r),
$$

and this is the precise disk of their convergence.

(ii) If $q$ satisfies $|(x-c) q|_{c}(r) \leq 1$, then the nontrivial solutions $u(x)$ to $L u=0$ near a $(c, r)$-generic point $t$ converge at least on the disk

$$
\operatorname{ord}(x-t)>\frac{1}{p-1}+\operatorname{ord}(t-c),
$$

and are bounded on this disk.

Proof. Let $u(x)$ be a solution to $L u=0$ which is analytic in a neighborhood of $t$, and which is normalized so that $u(t)=1$. Since $D u+q u=0$, we may define functions $\left\{b_{m}\right\}_{m \geq 0}$ such that $D^{m} u=b_{m} u$. We find that $b_{0}=1$, $b_{1}=-q, b_{2}=q^{2}-q^{\prime}$, and, in general, for $m>0$ the $b_{m}$ satisfy the recursion formula

$$
b_{m+1}=-q b_{m}+b_{m}^{\prime} .
$$

Since each $b_{m}$ is a polynomial in $q$ and its derivatives with integer coefficients, it follows that $b_{m} \in E_{c, r}$ for all $m$. Therefore, since $t$ is a $(c, r)$-generic point, we have $\left|b_{m}(t)\right|=\left|b_{m}\right|_{c}(r)$. By Taylor's theorem, in a neighborhood of $t$ we have

$$
u(x)=\sum_{m=0}^{\infty} \frac{b_{m}(t)}{m !}(x-t)^{m},
$$

since we assume $u(t)=1$. We will determine the radius of convergence of $u$ by computing $\left|b_{m}\right|_{c}(r)$.

First suppose that $q \in E_{c, r}$ satisfies $|(x-c) q|_{c}(r)>1$; we therefore have $|q|_{c}(r)>r^{-1}$. Since $b_{m} \in E_{c, r}$, Proposition 2.2 shows that $\left|b_{m}^{\prime}\right|_{c}(r) \leq$ $r^{-1}\left|b_{m}\right|_{c}(r)$, while on the other hand we have $\left|-q b_{m}\right|_{c}(r)>r^{-1}\left|b_{m}\right|_{c}(r)$ for all $m$. By applying induction to (3.5), we find that $\left|b_{m}\right|_{c}(r)=\left|q^{m}\right|_{c}(r)$ for all $m>0$. Therefore, from the Taylor expansion of $u$, it follows that $u(x)$ converges and is bounded on the disk given by (3.3), and that this is the exact domain of convergence for all solutions at $t$.

In the case where $|(x-c) q|_{c}(r) \leq 1$, a similar induction argument applied to (3.5) shows that $\left|b_{m}\right|_{c}(r) \leq r^{-m}$ for all $m$. It then follows from the Taylor expansion of $u$ that the solutions of $L$ at $t$ all converge on the disk (3.4) and 
are bounded on this disk; however, it is possible that they may converge on a larger disk.

Proof of Theorem 3.1. We proceed by induction on the order of $L$. We have already proven this theorem in the case where $n=1$ (Lemma 3.4). Suppose then that $n>1$ and assume that the theorem has been proven for all monic elements of $\mathfrak{R}_{c, r}$ of order less than $n$. Let $L \in \mathfrak{R}_{c, r}$ be as in the statement of the theorem; then if $u(x)$ is any solution to $L u=0$ which is analytic in a neighborhood of $t$, there are uniquely determined functions $\left\{b_{m}^{(j)}\right\}$ for $m \geq 0$ and $0 \leq j<n$ such that

$$
D^{m} u=b_{m}^{(n-1)} u^{(n-1)}+\cdots+b_{m}^{(1)} u^{\prime}+b_{m}^{(0)} u .
$$

(Here $u^{(j)}$ denotes $D^{j} u$, but $b_{m}^{(j)}$ need not denote $D^{j} b_{m}^{(0)}$.) We find that $b_{m}^{(j)}=\delta_{m, j}$ for $0 \leq m \leq n-1$, and that for all $m>0$ the functions $b_{m}^{(j)}$ satisfy the recursion relation

$$
\begin{aligned}
& b_{m+1}^{(j)}=-q_{n-j} b_{m}^{(n-1)}+b_{m}^{(j)^{\prime}}+b_{m}^{(j-1)} \quad(1 \leq j<n), \\
& b_{m+1}^{(0)}=-q_{n} b_{m}^{(n-1)}+b_{m}^{(0)^{\prime}} .
\end{aligned}
$$

From these formulae it is clear that each $b_{m}^{(j)} \in E_{c, r}$.

If every root $\lambda$ of $\Delta_{t}(L)$ satisfies $|\lambda| \leq r^{-1}$, it follows from the theory of Newton polygons that $\left|q_{j}\right|_{c}(r) \leq r^{-j}$ for all $j$. From (3.6) it is then easy to verify that $\left|b_{m}^{(j)}\right|_{c}(r) \leq r^{j-m}$ for all $j$ and $m$, using Proposition 2.2. Considering the Taylor expansion of $u$ at $t$ and using the definition of the $b_{m}^{(j)}$ we conclude that in this case every solution of $L$ at $t$ converges at least on the disk (3.2) and is bounded there. In this case, any optimal basis satisfies the conditions of the theorem. The theorem is therefore proven in this case.

For the remainder of this argument we will therefore suppose that $\Delta_{t}(L)$ has at least one root with absolute value larger than $r^{-1}$. We let $\gamma$ be a root of maximal modulus and let $\kappa$ be the number of roots of modulus $|\gamma|$; then by the theory of Newton polygons we find that

$$
\begin{aligned}
& \left|q_{\kappa}\right|_{c}(r)=|\gamma|^{\kappa}, \\
& \left|q_{j}\right|_{c}(r) \leq|\gamma|^{j} \quad \text { for } 1 \leq j \leq \kappa, \\
& \left|q_{j}\right|_{c}(r)<|\gamma|^{j} \quad \text { for } \kappa<j \leq n .
\end{aligned}
$$

From these inequalities, by applying induction to (3.6) one may easily verify that $\left|b_{m}^{(j)}\right|_{c}(r) \leq|\gamma|^{m-j}$ for all $m$ and $j$, and that $\left|b_{m}^{(j)}\right|_{c}(r)<|\gamma|^{m-j}$ for $j<n-\kappa$ when $m \geq n$. Therefore, every solution at $t$ converges at least on the disk $\operatorname{ord}(x-t)>1 /(p-1)+\log |\gamma|$, and every solution is bounded on this disk.

We now claim that the equality $\left|b_{m}^{(n-1)}\right|_{c}(r)=|\gamma|^{m+1-n}$ holds for infinitely many $m \in \mathbb{Z}^{+}$: First, we note that it holds for $m=n-1$. Now suppose that $m \geq n$ and that $\left|b_{m-1}^{(n-1)}\right|_{c}(r)=|\gamma|^{m-n}$ but $\left|b_{m}^{(n-1)}\right|_{c}(r)<|\gamma|^{m+1-n}$. Then by applying induction to (3.6), using (3.7) and the estimate $\left|b_{m}^{(j)}\right|_{c}(r)<|\gamma|^{m-j}$ for $j<n-\kappa$, we obtain $\left|b_{m}^{(n-\kappa)}\right|_{c}(r)=|\gamma|^{m+\kappa-n}$. Now let $j$ be minimal such that $\left|b_{m}^{(n-j)}\right|_{c}(r)=|\gamma|^{m+j-n} ;$ then $1<j \leq \kappa$. Then $(j-1)$-fold application of the recursion formulae (3.6) leaves us with

$$
\left|b_{n-1}^{m+j-1}\right|_{c}(r)=|\gamma|^{m+j-n},
$$

which verifies our claim. 
It follows that if $\varepsilon \in \Omega$ and $0<|\varepsilon|<|\gamma|$, then the set $\mathfrak{B}_{0}=\left\{v_{0}, \ldots, v_{n-1}\right\}$ of solutions at $t$ which are normalized by the conditions

$$
\begin{gathered}
v_{i}^{(j)}(t)=\delta_{i j} \varepsilon^{n-1-j} \quad \text { for } 0 \leq j<n-1, \\
v_{i}^{(n-1)}(t)=1 \quad \text { for } 0 \leq i \leq n-1
\end{gathered}
$$

is a basis of solutions at $t$ which all converge and are bounded exactly on the uncircumferenced disk given by $\operatorname{ord}(x-t)>1 /(p-1)+\log |\gamma|$.

This shows that at least one solution of $L$ at $t$ has $\operatorname{ord}(x-t)>1 /(p-1)+$ $\log |\gamma|$ as its exact disk of convergence. We now show that if $\Delta_{t}(L)$ has roots of absolute value less than $|\gamma|$, then $L$ has solutions with radii of convergence strictly greater than those in $\mathfrak{B}_{0}$.

Suppose that $0<\kappa<n$, where as before $\kappa$ is the number of roots of $\Delta_{t}(L)$ of absolute value $|\gamma|$, and all other roots have smaller absolute values. Then there exists $\sigma<|\gamma|$ such that

$$
\begin{aligned}
& \left|q_{\kappa}\right|_{c}(r)=|\gamma|^{\kappa}, \\
& \left|q_{i}\right|_{c}(r) \leq|\gamma|^{i} \text { for } 1 \leq i \leq \kappa, \\
& \left|q_{i}\right|_{c}(r) \leq|\gamma|^{\kappa} \sigma^{i-\kappa} \text { for } \kappa \leq i \leq n .
\end{aligned}
$$

We set $j_{0}=n-\kappa$, and note that $j_{0}>0$. Choose $\varrho$ such that $\varrho<|\gamma|$, $\varrho>r^{-1}$, and $\varrho>\sigma$. We will show that $L$ has solutions converging on the disk $\operatorname{ord}(x-t)>1 /(p-1)+\log \varrho$ by applying Lemma 3.2 with $\rho=|\pi| \varrho^{-1}$.

Suppose that $R \circ L=Q$ and write this equation explicitly in the form

$$
\begin{aligned}
& \left(g_{0} D^{m}+g_{1} D^{m-1}+\cdots+g_{m}\right) \circ\left(D^{n}+q_{1} D^{n-1}+\cdots+q_{n}\right) \\
& \quad=h_{0} D^{m+n}+h_{1} D^{m+n-1}+\cdots+h_{m+n},
\end{aligned}
$$

which gives the relations

$$
h_{m+n-k}=\sum_{\substack{l+j=k \\
0 \leq l \leq i \leq m \\
0 \leq j \leq n}}\left(\begin{array}{l}
i \\
l
\end{array}\right) g_{m-i} q_{n-j}^{(i-l)}
$$

for $0 \leq k \leq m+n$, with the convention $q_{0}=1$. In order to obtain a contradiction we assume that $\|Q-1\|_{\rho}<1$. This implies that $\left|h_{m+n}-1\right|_{c}(r)<1$, so $\left|h_{m+n}\right|_{c}(r)=1$. Now from (3.10) we have

$$
h_{m+n}=g_{m} q_{n}+g_{m-1} q_{n}^{\prime}+\cdots+g_{0} q_{n}^{(m)} .
$$

However, from (3.8) and Proposition 2.2 we also have

$$
\left|q_{n}^{(i)}\right|_{c}(r) \leq r^{-i}|i !||\gamma|^{n-j_{0}} \sigma^{j_{0}}<|\gamma|^{n-j_{0}} \varrho^{j_{0}+i}
$$

for $0 \leq i \leq m$ (the strict inequality holds for $i=0$ since $j_{0}>0$ ). So since $\left|h_{m+n}\right|_{c}(r)=1$, there must be an index $i, 0 \leq i \leq m$, such that $\left|g_{m-i}\right|_{c}(r)>$ $|\gamma|^{j_{0}-n} \varrho^{-j_{0}-i}$.

Let $i_{0}$ be any index, $0 \leq i_{0} \leq m$, such that the expression $\varrho^{i}\left|g_{m-i}\right|_{c}(r)$ attains its maximal value when $i=i_{0}$. Set $k_{0}=j_{0}+i_{0}$, and note that $k_{0}>0$ since $j_{0}>0$. By the result of the previous paragraph, we have $\left|g_{m-i_{0}}\right|_{c}(r)>$ $|\gamma|^{j_{0}-n} \varrho^{-k_{0}}$. We now proceed to show that $\|Q-1\|_{\rho} \geq 1$. 
From (3.10) we have

$$
h_{m+n-k_{0}}=\sum_{\substack{l+j=k_{0} \\
0 \leq l \leq i \leq m \\
0 \leq j \leq n}}\left(\begin{array}{l}
i \\
l
\end{array}\right) g_{m-i} q_{n-j}^{(i-l)} .
$$

Since $\sigma<\varrho<|\gamma|$ and $\varrho>r^{-1}$, equations (3.8), Proposition 2.2, and the definition of $j_{0}$ together imply that in each term in the sum (3.11), the factor $q_{n-j}^{(i-l)}$ satisfies

$$
\left|q_{n-j}^{(i-l)}\right|_{c}(r)<\varrho^{i-i_{0}}\left|q_{n-j_{0}}\right|_{c}(r),
$$

except for the term in which $i_{0}=i=l$, in which case we have the trivial equality. Then by considering the definition of $i_{0}$, we find that each term in the sum (3.11) for $h_{m+n-k_{0}}$ satisfies

$$
\left|\left(\begin{array}{l}
i \\
l
\end{array}\right) g_{m-i} q_{n-j}^{(i-l)}\right|_{c}(r)<\left|g_{m-i_{0}} q_{n-j_{0}}\right|_{c}(r),
$$

except for the term $g_{m-i_{0}} q_{n-j_{0}}$ itself.

Therefore we have $\left|h_{m+n-k_{0}}\right|_{c}(r)=\left|g_{m-i_{0}} q_{n-j_{0}}\right|_{c}(r)$. From the definitions of $i_{0}$ and $j_{0}$ we find $\left|h_{m+n-k_{0}}\right|_{c}(r)>\varrho^{-k_{0}}$. Since $m+n-k_{0} \neq m+n$, for $\rho=|\pi| \varrho^{-1}$ we have

$$
\|Q-1\|_{\rho}>\left|k_{0} !\right||\pi|^{-k_{0}} \varrho^{k_{0}}\left|h_{m+n-k_{0}}\right|_{c}(r)>1 .
$$

This obviously contradicts the assumption that $\|Q-1\|_{\rho}<1$. Therefore, by Lemma 3.2, there exist solutions of $L$ at $t$ which converge at least on the disk $\operatorname{ord}(x-t)>1 /(p-1)+\log \varrho$.

Thus we have shown that if $\Delta_{t}(L)$ has roots of absolute value less than $|\gamma|$, then $L$ has solutions at $t$ with radii of convergence strictly greater than those in $\mathfrak{B}_{0}$. Therefore, if $L$ has no solutions at $t$ which have greater radius of convergence than those in $\mathfrak{B}_{0}$, then $\mathfrak{B}_{0}$ is an optimal basis and every root of $\Delta_{t}(L)$ has absolute value equal to $|\gamma|$. The theorem is therefore proven in the case where $\mathfrak{B}_{0}$ is an optimal basis.

We will now show that if $\mathfrak{B}_{0}$ is not an optimal basis, then $\Delta_{t}(L)$ has roots of absolute value less than $|\gamma|$. For this we will need to use the induction hypothesis. Suppose that there exist solutions of $L$ at $t$ which converge on a disk which properly contains the circumferenced disk ord $(x-t) \geq 1 /(p-1)+\log |\gamma|$. Then by Lemma 3.3 there is a monic right factor $M$ of $L$ (with coefficients in $\left.E_{c, r}\right)$ which annihilates precisely those solutions. By our hypotheses, if $\nu$ is the order of $M$ then $0<\nu<n$.

We write the equation $N \circ M=L$ more explicitly in the form

$$
\begin{aligned}
& \left(D^{n-\nu}+g_{1} D^{n-\nu-1}+\cdots+g_{n-\nu}\right) \circ\left(D^{\nu}+f_{1} D^{\nu-1}+\cdots+f_{\nu}\right) \\
& \quad=D^{n}+q_{1} D^{n-1}+\cdots+q_{n},
\end{aligned}
$$

from which we may deduce the relations

$$
q_{n-k}=\sum_{\substack{l+j=k \\
0 \leq l \leq i \leq n-\nu \\
0 \leq j \leq \nu}}\left(\begin{array}{l}
i \\
l
\end{array}\right) g_{n-\nu-i} f_{\nu-j}^{(i-l)}
$$


for $0 \leq k \leq n$. (Here we use $q_{0}=g_{0}=f_{0}=1$ as convention.) Since $\nu<n$, we may apply the induction hypothesis to $M$ to conclude that every root of $\Delta_{t}(M)$ has absolute value less than $|\gamma|$. It follows that $\left|f_{j}\right|_{c}(r)<|\gamma|^{j}$ for $1 \leq j \leq \nu$. Therefore, in equation (3.13), the expressions $f_{\nu-j}^{(i-l)}$ in the terms of the sum for $q_{n-k}$ all satisfy

$$
\left|f_{\nu-j}^{(i-l)}\right|_{c}(r)<|\gamma|^{i+\nu-k}
$$

except when $j=\nu$ and $i=l$, in which case we have the trivial equality $f_{0}^{(0)}=1$.

We first proceed to show that $\left|g_{i}\right|_{c}(r) \leq|\gamma|^{i}$ for $0 \leq i \leq n-\nu$. Supposing this does not hold, let $i$ be maximal such that $\left|g_{n-\nu-i}\right|_{c}(r)>|\gamma|^{n-\nu-i}$. From (3.13), we see that for $j=\nu, l=i$, and $k=i+\nu$, we have $g_{n-\nu-i}$ as a term in the sum for $q_{n-k}$, and by (3.14) we find that all other terms in this sum have strictly smaller absolute value. Thus $\left|q_{n-k}\right|_{c}(r)=\left|g_{n-\nu-i}\right|_{c}(r)>|\gamma|^{n-k}$, contradicting (3.7). Therefore we must have $\left|g_{i}\right|_{c}(r) \leq|\gamma|^{i}$ for all $i$.

Having defined $\nu>0$ to be the order of $M$, and $\kappa$ to be the number of roots of $\Delta_{t}(L)$ of absolute value $|\gamma|$, we set $\mu=n-\kappa$. We will now show that $\mu=\nu$.

Suppose $0 \leq k<\nu$. Then every term in the sum (3.13) for $q_{n-k}$ must have $j<\nu$, so by (3.14) in these terms we have $\left|f_{\nu-j}^{(i-l)}\right|_{c}(r)<|\gamma|^{i+\nu-k}$. From our estimate for $\left|g_{i}\right|_{c}(r)$ we then find that $\left|q_{n-k}\right|_{c}(r)<|\gamma|^{n-k}$. Then (3.7) shows that $k \neq \mu$. Thus $\mu$ is not less than $\nu$.

Suppose $k \geq \nu$. Then the terms in the sum (3.13) for $q_{n-k}$ all satisfy

$$
\left|\left(\begin{array}{l}
i \\
l
\end{array}\right) g_{n-\nu-i} f_{\nu-j}^{(i-l)}\right|_{c}(r) \leq|\gamma|^{n-k}
$$

with equality holding if and only if $\nu=j, i=l$, and $\left|g_{n-\nu-i}\right|_{c}(r)=|\gamma|^{n-\nu-i}=$ $|\gamma|^{n-k}$. Since $\left|q_{n-\mu}\right|_{c}(r)=|\gamma|^{n-\mu}$ and $\mu$ is minimal with this property, it follows that $\left|g_{n-\mu}\right|_{c}(r)=|\gamma|^{n-\mu}$ and $\mu$ is also minimal with this property.

Suppose then that $\mu>\nu$. We have $\left|g_{n-\mu}\right|_{c}(r)=|\gamma|^{n-\mu}$, and $\left|g_{n-k}\right|_{c}(r)<$ $|\gamma|^{n-k}$ for $\nu \leq k<\mu$. Then $\Delta_{t}(N)$ has $n-\mu$ roots of absolute value $|\gamma|$ and $\mu-\nu$ roots of smaller absolute value. By applying the induction hypothesis to $N$, we find that there is exactly a $(\mu-\nu)$-dimensional subspace of solutions to $N u=0$ which converge on a disk which properly contains the circumferenced disk $\operatorname{ord}(x-t) \geq 1 /(p-1)+\log |\gamma|$. It follows from Lemma 3.3 that $N$ factors in $\mathfrak{R}_{c, r}$ as $N=N_{2} \circ N_{1}$, where $N_{1}$ annihilates precisely those solutions of $N$ converging on a disk properly containing the disk ord $(x-t) \geq 1 /(p-1)+\log |\gamma|$, and so is of order $\mu-\nu$. But then by the induction hypothesis the roots of $\Delta_{t}\left(N_{1}\right)$ are all of absolute value strictly less than $|\gamma|$. Thus we have $L=$ $N_{2} \circ\left(N_{1} \circ M\right)$, where $N_{1} \circ M$ is of order $\mu$; it is also easy to verify, by relations similar to (3.13), that all the roots of $\Delta_{t}\left(N_{1} \circ M\right)$ have absolute value less than $|\gamma|$, using the fact that $N_{1}$ and $M$ each have this property. Therefore $N_{1} \circ M$ (and hence $L$ ) has a $\mu$-dimensional space of solutions converging on a disk properly containing ord $(x-t) \geq 1 /(p-1)+\log |\gamma|$, contradicting the definition of $M$ and $\nu$. Therefore $\mu=\nu$, and $\left|g_{n-\nu}\right|_{c}(r)=|\gamma|^{n-\nu}$.

We are now able to construct the one-to-one correspondence described in the theorem. We recall that the solutions of $M$ at $t$ are precisely the solutions of 
$L$ at $t$ which converge on a disk which properly contains the disk ord $(x-t)$ $\geq 1 /(p-1)+\log |\gamma|$, and that our induction hypothesis states that the theorem holds for $M$. Therefore let $\mathfrak{B}_{1}=\left\{u_{1}, \ldots, u_{\nu}\right\}$ be any optimal basis for the kernel of $M$ at $t$ which satisfies the conditions of the theorem for $M$. It follows from the definitions of $\mathfrak{B}_{0}$ and of $M$ that $\mathfrak{B}_{1}$ may be extended to form an optimal basis $\mathfrak{B}$ for the kernel of $L$ at $t$ by including $n-\nu$ elements of $\mathfrak{B}_{0}$, chosen so that $\mathfrak{B}$ remains independent. To establish the correspondence for $L$, we first require that the $n-\nu$ roots of $\Delta_{t}(L)$ of absolute value $|\gamma|$ correspond to the $n-\nu$ elements of $\mathfrak{B}$ which were adjoined from $\mathfrak{B}_{0}$. Since $|\gamma|>r^{-1}$ and the elements of $\mathfrak{B}_{0}$ all have ord $(x-t)>1 /(p-1)+\log |\gamma|$ as their exact disk of convergence, the conditions of the correspondence are satisfied for the roots of $\Delta_{t}(L)$ of magnitude $|\gamma|$. Finally, since the correspondence between the roots of $\Delta_{t}(M)$ and the elements of $\mathfrak{B}_{1}$ satisfies the required condition by the induction hypothesis, to complete the proof of the theorem for $L$ it suffices to show that the absolute values of the roots of $\Delta_{t}(M)$ are exactly the same as the absolute values of the roots of $\Delta_{t}(L)$ which are smaller than $|\gamma|$.

Suppose then that $\Delta_{t}(M)$ has exactly $l$ roots of absolute value $\sigma$. Then $\sigma<|\gamma|$, and by the theory of Newton polygons there exists an integer $j$ with $0 \leq j-l<j \leq \nu$ such that

$$
\begin{aligned}
& \left|f_{\nu-j}\right|_{c}(r)=\Lambda \geq \sigma^{\nu-j}, \\
& \left|f_{\nu+l-j}\right|_{c}(r)=\Lambda \cdot \sigma^{l}, \\
& \left|f_{\nu-i}\right|_{c}(r) \leq \Lambda \cdot \sigma^{j-i} \quad \text { for } j-l \leq i \leq j, \\
& \left|f_{\nu-i}\right|_{c}(r)<\Lambda \cdot \sigma^{j-i} \quad \text { for } 0 \leq i<j-l \text { or } \quad j<i \leq \nu .
\end{aligned}
$$

Then by applying (3.13) we find that

$$
\begin{array}{ll}
\left|q_{n-j}\right|_{c}(r)=\left|g_{n-\nu} f_{\nu-j}\right|_{c}(r)=\Lambda \cdot|\gamma|^{n-\nu}, & \\
\left|q_{n+l-j}\right|_{c}(r)=\left|g_{n-\nu} f_{\nu+l-j}\right|_{c}(r)=\Lambda \cdot|\gamma|^{n-\nu} \sigma^{l}, \\
\left|q_{n-i}\right|_{c}(r) \leq\left|g_{n-\nu} f_{\nu-i}\right|_{c}(r) \leq \Lambda \cdot|\gamma|^{n-\nu} \sigma^{j-i} & \text { for } j-l \leq i \leq j, \\
\left|q_{n-i}\right|_{c}(r) \leq\left|g_{n-\nu} f_{\nu-i}\right|_{c}(r)<\Lambda \cdot|\gamma|^{n-\nu} \sigma^{j-i} & \text { for } 0 \leq i<j-l \\
& \text { or } j<i \leq \nu .
\end{array}
$$

Finally, if $\nu<i \leq n$, then since $\Lambda \geq \sigma^{\nu-j}$ and $\left|q_{n-i}\right|_{c}(r) \leq|\gamma|^{n-i}$, we may write

$$
\left|q_{n-i}\right|_{c}(r)<\Lambda \cdot|\gamma|^{n-\nu} \sigma^{j-i} \text {. }
$$

By the theory of Newton polygons, equations (3.16) and (3.17) imply that $\Delta_{t}(L)$ has exactly $l$ roots with absolute value equal to $\sigma$, as desired. Therefore, we have proven that $L$ satisfies the conditions of the theorem. By induction, the theorem holds for all monic elements of $\mathfrak{R}_{c, r}$, and the proof is complete.

Remarks. Although all optimal bases for the kernel of $L$ at $t$ have corresponding solutions with the same radii of convergence, in the above proof we have taken care to select a particular optimal basis $\mathfrak{B}$ so that the elements of $\mathfrak{B}$ which do not converge for $\operatorname{ord}(x-t)>1 /(p-1)+\operatorname{ord}(t-c)$ have uncircumferenced disks as their exact disks of convergence. We do not know whether the conditions of this theorem hold for every optimal basis $\mathfrak{B}$; in particular, we know of no example of a linear differential operator having a solution at a 
generic point whose disk of convergence is circumferenced. It should be noted that if the absolute values of the roots of $\Delta_{t}(L)$ which are larger than $r^{-1}$ are all distinct, then every optimal basis for the kernel of $L$ at $t$ has the required property.

In [13] we have also shown that indeed every optimal basis for the kernel of $L$ at $t$ does satisfy the condition of this theorem when the operator $L$ is of order two. Therefore, we can conclude that the disk of convergence is uncircumferenced for all solutions at $t$ which do not converge on the disk (3.2) when the order of the operator is two or less. We conjecture that this is true for all $L \in \mathfrak{R}_{c, r}$.

The alternate method of proof for operators of order two given in [13] is based on an analysis of the continued fraction expansion for a solution to the Riccati equation associated to a second-order linear operator (cf. [6, §7.5]). There we showed that if $L$ is of order two and $\Delta_{t}(L)$ has roots of distinct absolute value with at least one root larger than $r^{-1}$, then the larger of the two radii of convergence is determined exactly by the rate at which the continued fraction converges to a solution of the Riccati equation. Unfortunately this method does not readily extend to operators of order greater than two.

\section{Applications}

We first combine the results of Theorem 3.1 and Lemma 3.3 and rephrase them in terms of the Newton polygon of $\Delta_{t}(L)$, showing the complete relationship between the factorization of the type described by Lemma 3.3 and factorization according to the slopes of the associated Newton polygon (cf. [5, $\S 6.2 .3 .3])$.

Corollary 4.1. Let $L$ be a monic element of $\mathfrak{R}_{c, r}$ and let $t$ be a $(c, r)$-generic point. Suppose that $m_{k}<m_{k-1}<\cdots<m_{1}$ are the slopes of the Newton polygon of $\Delta_{t}(L)$ and that the side of slope $m_{i}$ has horizontal length of projection $n_{i}$ for $1 \leq i \leq k$; suppose further that $m_{i}>-\log r$ for $1 \leq i \leq j$, and set $n^{\prime}=n_{j+1}+\cdots+n_{k}$. Then there exist monic elements $L_{1}, \ldots, L_{j}, L^{\prime}$ of $\Re_{c, r}$ of orders $n_{1}, \ldots, n_{j}, n^{\prime}$, respectively, such that

$$
L=L_{1} \circ \cdots \circ L_{j} \circ L^{\prime} \text {. }
$$

Furthermore, $L^{\prime}$ annihilates precisely those solutions of $L$ at $t$ which converge at least on the disk $\operatorname{ord}(x-t)>1 /(p-1)+\operatorname{ord}(t-c)$, and for $1 \leq i \leq j$ the product $L^{(i)}=L_{i} \circ \cdots \circ L_{j} \circ L^{\prime}$ annihilates precisely those solutions of $L$ at $t$ which converge at least on the disk

$$
\operatorname{ord}(x-t)>\frac{1}{p-1}+m_{i}
$$

Proof. The conditions on the Newton polygon of $\Delta_{t}(L)$ imply that $\Delta_{t}(L)$ has precisely $n_{i}$ roots of absolute value $p^{m_{i}}$ for each $i$. Since the absolute values of the roots corresponding to the slopes $m_{j+1}, \ldots, m_{k}$ are no larger than $r^{-1}$, we see from Theorem 3.1 that there is exactly an $n^{\prime}$-dimensional space of solutions converging at least on the disk (3.2). By Lemma 3.3 there is a unique monic right factor $L^{\prime}$ of $L$ (of order $n^{\prime}$ ) which annihilates precisely those solutions. Applying Theorem 3.1 to the remaining roots of $\Delta_{t}(L)$ shows that for $1 \leq i \leq j$, the space of solutions converging at least for $\operatorname{ord}(x-t)>1 /(p-1)+m_{i}$ has dimension $n_{i}+\cdots+n_{j}+n^{\prime}$. Then by Lemma 3.3 there is a unique monic right 
factor $L^{(i)}$ of $L$ (of order $n_{i}+\cdots+n_{j}+n^{\prime}$ ) which annihilates precisely these solutions. From the uniqueness statement we see that $L^{\prime}$ is a right factor of each $L^{(i)}$, and that $L^{\left(i_{2}\right)}$ is a right factor of $L^{\left(i_{1}\right)}$ whenever $1 \leq i_{1}<i_{2} \leq j$. This gives the required factorization.

We now connect the above results on radii of convergence with cerain properties of the index of a differential operator to give a partial proof of the indexradius conjecture of Robba.

Proposition 4.2. Let $L=g_{0} D^{n}+g_{1} D^{n-1}+\cdots+g_{n} \in \mathfrak{G}_{A}$, where $A$ is a $(c, r)$ very standard set. Suppose that every root $\lambda$ of $\Delta_{t}(L)$ satisfies $|\lambda|>r^{-1}$. Then $L$ is injective and has an index as an operator on $H(A)$, and that index is given by the formula

$$
\chi(L, H(A))=-\operatorname{ord}_{A}\left(g_{n}\right) .
$$

Proof. We note that the condition on the roots of $\Delta_{t}(L)$ implies that $\left\|g_{n}\right\|_{c, r}>$ $\left\|L-g_{n}\right\|_{c, r}$ as operators on $H(A)$, by the theory of Newton polygons. It then follows from Lemma 4.4 of [8] and Proposition 2.1 that $L$ is injective and that

$$
\chi(L, H(A))=\chi\left(g_{n}, H(A)\right)=-\operatorname{ord}_{A}\left(g_{n}\right) .
$$

Corollary 4.3. Let $A=B\left(c, R^{+}\right)$and let $L=g_{0} D^{n}+g_{1} D^{n-1}+\cdots+g_{n} \in \mathfrak{G}_{A}$. Suppose that, for some $r_{0}<R$, every root $\lambda$ of $\Delta_{t_{0}}(L)$ (where $t_{0}$ is a $\left(c, r_{0}\right)$ generic point) satisfies $|\lambda|>r_{0}^{-1}$. Then the formula

$$
\left(\frac{d \log \rho_{c}(L, r)}{d \log r}\right)^{ \pm}=\chi_{c}^{ \pm}(L, r)+\operatorname{ord}_{c}^{ \pm}\left(g_{0}, r\right)
$$

(conjectured by Robba in [10]) holds for $L$ and for all $r \in\left|\Omega^{*}\right|$ sufficiently close to $r_{0}$.

Proof. It follows from Theorem 3.1 that for all $r \in\left|\Omega^{*}\right|$ sufficiently close to $r_{0}$ we have

$$
\rho_{c}(L, r)=\prod_{i=1}^{n}\left|\frac{\pi}{\lambda_{i}}\right|=|\pi|^{n}\left|\frac{g_{0}}{g_{n}}\right|_{c}(r)
$$

(where $\lambda_{1}, \ldots, \lambda_{n}$ are the roots of $\Delta_{t}(L), t$ being a $(c, r)$-generic point), so from the well-known relation [1, Proposition 4.3.2]

$$
\left(\frac{d \log |f|_{c}(r)}{d \log r}\right)^{ \pm}=\operatorname{ord}_{c}^{ \pm}(f, r),
$$

we may deduce that

$$
\begin{aligned}
\left(\frac{d \log \rho_{c}(L, r)}{d \log r}\right)^{ \pm} & =\left(\frac{d \log \left|g_{0} / g_{n}\right|_{c}(r)}{d \log r}\right)^{ \pm}=\operatorname{ord}_{c}^{ \pm}\left(\frac{g_{0}}{g_{n}}, r\right) \\
& =\operatorname{ord}_{c}^{ \pm}\left(g_{0}, r\right)-\operatorname{ord}_{c}^{ \pm}\left(g_{n}, r\right) .
\end{aligned}
$$

But by Proposition 4.2 we find that for such $r$,

$$
\chi_{c}^{ \pm}(L, r)=-\operatorname{ord}_{c}^{ \pm}\left(g_{n}, r\right),
$$

from which the corollary follows.

Formula (4.1) was proven by Robba for operators of order one [10], and it was conjectured that this formula should hold for operators of any order provided 
the operator has no solution at a $\left(c, r_{0}\right)$-generic point $t$ which converges for $\operatorname{ord}(x-t)>\operatorname{ord}(t-c)$. The above corollary asserts that the formula holds under the stronger assumption that there is no solution converging for $\operatorname{ord}(x-t)>$ $1 /(p-1)+\operatorname{ord}(t-c)$; this condition is equivalent to the condition that all roots $\lambda$ of $\Delta_{t}(L)$ satisfy $|\lambda|>r_{0}^{-1}$, by Theorem 3.1 .

As further applications of Theorem 3.1 we present two results concerning the description of the solutions of linear differential operators.

Corollary 4.4. Let $L \in \mathfrak{R}_{c, r}$, and let $t$ be a $(c, r)$-generic point. If $L$ has an unbounded solution at $t$ then that solution converges on an uncircumferenced disk which properly contains the disk

$$
\operatorname{ord}(x-t)>\frac{1}{p-1}+\operatorname{ord}(t-c) .
$$

Proof. From Theorem 3.1(i) we see that solutions at $t$ which do not converge on the disk (4.2) are all bounded. Furthermore, if a solution converges at least on the disk (4.2), it is bounded on this disk by Theorem 3.1(ii); therefore such a solution must converge on a strictly larger disk. But if an analytic function is unbounded, then its disk of convergence must be uncircumferenced.

Corollary 4.5. Let $L \in \mathfrak{R}_{c, r}$, and let $t$ be a $(c, r)$-generic point. For $\rho \in \mathbb{R}^{+}$ let $\delta(t, \rho)$ be the dimension over $\Omega$ of the space of solutions of $L u=0$ which converge at least on the disk

$$
\operatorname{ord}(x-t)>\frac{1}{p-1}+\log \rho \text {. }
$$

If furthermore $L \in \mathfrak{R}_{A}$ for some $(c, r)$-very standard set $A$ which contains $B\left(c, r^{-}\right)$, let $\gamma_{r}$ be the dimension over $\Omega$ of the space of solutions of $L u=0$ which are analytic on $B\left(c, r^{-}\right)$.

(i) For $\rho \geq r^{-1}, \delta(t, \rho)$ is equal to the number of roots $\lambda$ of $\Delta_{t}(L)$ which satisfy $|\lambda| \leq \rho$.

(ii) For $L \in \mathfrak{R}_{A}$ as above, $\gamma_{r} \leq \delta\left(t, r^{-1}\right)$.

Proof. The first result follows immediately from Theorem 3.1. For (ii), first suppose that $u$ is a solution which is analytic on $B\left(c, r^{-}\right)$. Then whenever $t_{0}$ is a $\left(c, r_{0}\right)$-generic point with $r_{0}<r$, we know that $u$ converges on $B\left(t_{0}, r_{0}^{-}\right)$. Viewing the coefficients of $L$ as elements of $M\left(B\left(c, r_{0}^{-}\right)\right)$, by Theorem 3.1 there is a corresponding root $\lambda_{0}$ of $\Delta_{t_{0}}(L)$ with $\left|\lambda_{0}\right| \leq r_{0}^{-1}$. It follows that $\gamma_{r} \leq \delta\left(t_{0}, r_{0}^{-1}\right)$ for all $\left(c, r_{0}\right)$-generic points $t_{0}$ with $r_{0}<r$. Finally, the theory of Newton polygons implies that if $\Delta_{t_{0}}(L)$ has exactly $j$ roots of absolute value at most $r_{0}^{-1}$ then $\left|q_{n-i} / q_{n-j}\right|_{c}\left(r_{0}\right) \leq r_{0}^{i-j}$ for $0 \leq i \leq j$. Since $|h|_{c}\left(r_{0}\right)$ is a continuous function of $r_{0} \in(0, r]$ for $h \in M(A)$, we find that $\gamma_{r} \leq \delta\left(t_{0}, r_{0}^{-1}\right)$ for all $r_{0}<r$ implies that $\gamma_{r} \leq \delta\left(t, r^{-1}\right)$ as well, giving (ii). This completes the proof.

We note that part (i) above implies that, if $L \in \mathfrak{G}_{A}$ is completely soluble in the generic disk $B\left(t, r^{-}\right)$, then every root $\lambda$ of $\Delta_{t}(L)$ satisfies $|\lambda| \leq r^{-1}$. Furthermore, if every root $\lambda$ of $\Delta_{t}(L)$ satisfies $|\lambda|>r^{-1}$, then $L$ is completely insoluble in $B\left(t, r^{-}\right)$.

We present the following application to the study of solutions at irregular singular points as an extension of the idea in part (ii) above. Here we give a $p$-adic proof and extension of a classical result. 
Corollary 4.6. Let $L=D^{n}+q_{1} D^{n-1}+\cdots+q_{n} \in \mathfrak{R}_{A}$, where $A$ contains a disk about the point $c \in K$; for $0 \leq j \leq n$ let $\varpi_{j}=-\operatorname{ord}_{c} q_{j}$ denote the order of the pole of $q_{j}$ at $x=c$, with $\varpi_{0}=0$. Assume that $L$ has an irregular singularity at $x=c$; thus $\varpi_{j}-j>0$ for some $j$. Let $k$ be the minimal integer with the following properties:

(i) The difference $\varpi_{j}-j$ attains its maximal value when $j=k$.

(ii) Among those indices $j$ for which the above maximum is attained, the expression

$$
\left|\lim _{x \rightarrow c}(x-c)^{\varpi_{j}} q_{j}(x)\right|
$$

attains its maximal value when $j=k$.

Then the space of solutions of $L u=0$ which are analytic near $x=c$ has dimension at most $n-k$ over $\Omega$.

Proof. The hypotheses on $L$ and the definition of $k$ imply that, for all sufficiently small $r \in\left|\Omega^{*}\right|$, we have $\left|q_{k}\right|_{c}(r)>r^{-k}$ and $\left|q_{k} / q_{j}\right|_{c}(r)>r^{j-k}$ for all $j<k$. It follows that $\Delta_{t}(L)$ has at least $k$ roots of absolute value greater than $r^{-1}$ for all sufficiently small $r$. By Theorem 3.1, for small $r$ there is at most an $(n-k)$-dimensional space of solutions which can converge in $B\left(t, r^{-}\right)$. Since any solution $u$ which is analytic at $x=c$ converges in all $(c, r)$-generic disks when $r$ is less than the radius of convergence of $u$ at $x=c$, the result follows.

This result is an analogue of a well-known principle of operators with irregular singular points in the complex domain (e.g., [6, $§ 17.11]$ ). If the coefficients of $L$ are meromorphic and $c \in \mathbb{C}$, then the class $k^{\prime}$ of the irregular singular point $x=c$ is defined to be the least integer which satisfies condition (i) in the statement of the above corollary. Then it is known that $L u=0$ has at most $n-k^{\prime}$ independent complex regular solutions at $x=c$. This corollary implies that if $L \in \overline{\mathbb{Q}}(x)[D]$ then this classical result is true with $k^{\prime}$ replaced by the integer $k$ (defined above) associated to any $p$-adic imbedding of $\overline{\mathbb{Q}}$. The interpretation is that, while $n-k^{\prime}$ is the degree of the classical indicial polynomial of $L$ at $x=c, n-k$ is a sharper upper bound for the number of roots of the indicial polynomial which can be $p$-integral.

We conclude this article with a specific example. Here we use Theorem 3.1 to explain and analyze the phenomenon of distinct radii of convergence of solutions of the second-order operator which annihilates the confluent hypergeometric function.

Example 4.7. Consider the differential operator

$$
L_{a}=D^{2}-\frac{x}{p(1-x)} D-\frac{a}{p(1-x)},
$$

where $a \in K$ satisfies $|a| \leq 1$. It has been noted by Monsky that this operator has solutions with distinct radii of convergence at a generic point $t$ satisfying $|t-1|=1$ when $a \in \mathbb{Z}_{p}$ is not a negative integer. This phenomenon has been treated by Robba and Dwork in $[8,5]$, using the fact that for such values of $a$, the confluent hypergeometric function

$$
{ }_{1} F_{1}\left(a, \frac{1}{p} ; \frac{(1-x)}{p}\right)=\sum_{m=0}^{\infty} \frac{(a)_{m}}{(1 / p)_{m} m !}\left(\frac{1-x}{p}\right)^{m}
$$


is a solution of $L$ near $x=1$ which converges in $B\left(1,1^{-}\right)$, so there is also a solution which converges and is bounded in $B\left(t, 1^{-}\right)$; however, the Wronskian at $t$ does not converge on $B\left(t, 1^{-}\right)$. We will use Theorem 3.1 to show that $L_{a}$ has solutions with distinct radii of convergence at all generic points $t$ for $c=1$ under the weaker hypothesis that $|a| \leq 1$.

Let $t$ be a $(1, r)$-generic point and let $\lambda, \sigma$ be the roots of $\Delta_{t}(L)$, with $|\lambda| \geq|\sigma|$. When $r \leq 1$ we have $|\lambda|=p r^{-1},|\sigma|=|a|$, while for $r \geq 1$ we have $|\lambda|=p,|\sigma|=|a| r^{-1}$. We find from Theorem 3.1 that for all $r$, there is a one-dimensional space of solutions at $t$ which converge at least for

$$
\operatorname{ord}(x-t)>\frac{1}{p-1}+\operatorname{ord}(t-1)
$$

and are bounded on that disk; the remaining solutions converge and are bounded exactly on the disk

$$
\operatorname{ord}(x-t)>\frac{p}{p-1}+\operatorname{ord}(t-1) \quad\left(\text { resp. } \operatorname{ord}(x-t)>\frac{p}{p-1}\right)
$$

when $r \leq 1$ (resp. $r \geq 1$ ). Therefore for any $a \in K \cap B\left(0,1^{+}\right)$, the operator $L_{a}$ has solutions with distinct radii of convergence at every $(1, r)$-generic point, regardless of the radius $r$. We therefore consider the existence of solutions with distinct radii of generic convergence an analytic property of $L_{a}$ (depending only on the norms of the coefficients), although for $r=1$ the existence of a solution converging in $B\left(t, 1^{-}\right)$is an algebraic property of $L_{a}$ when $a \in \mathbb{Z}_{p}$.

We remark that the convergence behavior of $L_{a}$ is slightly different when $|a|>1$. If we let $|a|=p$, for example, then for $r \leq 1$ we have $|\lambda|=p r^{-1}$, $|\sigma|=p$, while for $r \geq 1$ we have $|\lambda|=|\sigma|=p r^{-1 / 2}$. So for $r<p^{-1}$ there are solutions at $t$ with ordinals of convergence

$$
\frac{p}{p-1}+\operatorname{ord}(t-1) \text { and at least } \frac{1}{p-1}+\operatorname{ord}(t-1) \text {, }
$$

respectively; for $p^{-1} \leq r<1$ the ordinals of convergence are

$$
\frac{p}{p-1}+\operatorname{ord}(t-1) \text { and } \frac{p}{p-1},
$$

respectively; but for $r \geq 1$ all solutions have the same ordinal of convergence

$$
\frac{p}{p-1}+\frac{1}{2} \operatorname{ord}(t-1) \text {. }
$$

In particular, for $r<1$, the radii of convergence of solutions at $(1, r)$-generic points $t$ are distinct, while for $r \geq 1$ all solutions have the same radius of convergence.

\section{REFERENCES}

1. Y. Amice, Les nombres p-adiques, Presses Univ. de France, 1975.

2. G. Christol, Modules différentiels et equations différentielles p-adiques, Queen's Papers in Pure and Appl. Math., Queen's Univ., Kingston, Ont., 1983.

3. D. N. Clark, A note on the p-adic convergence of solutions of linear differential equations, Proc. Amer. Math. Soc. 17 (1966), 262-269.

4. B. Dwork, On p-adic differential equations. II, Ann. of Math. (2) 98 (1973), 366-376. 
5. B. Dwork and P. Robba, On ordinary linear p-adic differential equations, Trans. Amer. Math. Soc. 231 (1977), 1-46.

6. E. L. Ince, Ordinary differential equations, Dover, New York, 1944.

7. B. Malgrange, Sur les points singuliers des équations différentielles, Enseign. Math. (2) 20 (1974), 147-176.

8. P. Robba, On the index of p-adic differential operators. I, Ann. of Math. (2) 101 (1975), 280-316.

9. __ On the index of p-adic differential operators. II, Duke Math. J. 43 (1976), 19-31.

10. __ On the index of p-adic differential operators. III. Application to twisted exponential sums, Astérisque 119-120 (1984), 191-266.

11. __ Indice d'un opérateur différentiel p-adique. IV. Cas des systèmes, Ann. Inst. Fourier 35 (1985), 13-55.

12. _ـ Symmetric powers of the p-adic Bessel equation, J. Reine Angew. Math. 366 (1986), 194-220.

13. P. T. Young, Ph.D. Dissertation, Oklahoma State University, 1988.

Department of Mathematics, College of Charleston, Charleston, South Carolina 29424

E-mail address: youngp@citadel.bitnet 